\title{
Yeast Hyphae Screening
}

National Cancer Institute

\section{Source}

National Cancer Institute. Yeast Hyphae Screening. NCI Thesaurus. Code C92239.

Examination of a biological specimen to detect the presence of any yeast cells that are in the long, filamentous and branching phase of growth. 